examined by determination of sphere-forming capacities, the frequency of sphere-forming and tumor-initiating cells and the expression levels of OCSCs markers. Immunoprecipitation assay was used for determination of the binding between PARP1 and nuclear YAP. The post-translational modification of YAP was examined by western blot.

Results PARP1 positively regulates the stemness of OCSCs; treatment with PARP1 inhibitors also suppressed the stemness of OCSCs. PARP1 enhanced YAP activity reflected by upregulation of the mRNA levels of YAP target genes and the protein level of nuclear YAP. PARP1 enhanced the stabilization of YAP in nucleus and the ubiquitination of nuclear YAP was involved in this process. The positive correlation between PARP1 and nuclear YAP was found in both cell-based and mice models. Knockdown of YAP abolished the effect of PARP1 on OCSCs stemness.

Conclusions PARP1 promotes the stemness of OCSCs and stabilization of nuclear YAP is the underlying mechanism. This finding is useful for extension the clinical use of PARP1 inhibitors for eradicating human OCSCs.

\section{EPV217/\#534 IMPACT OF RESIDUAL TUMOR SIZE BY COMPUTED TOMOGRAPHY AFTER PRIMARY OPTIMAL CYTOREDUCTION ON PROGNOSIS OF ADVANCED OVARIAN CANCER}

${ }^{1} \mathrm{H}$ Lim*, 'SJ Park, ${ }^{1} E J$ Lee, ${ }^{1} \mathrm{M}$ Lee, ${ }^{2}$ SY Kim, ${ }^{1} \mathrm{HH}$ Chung, ${ }^{1} \mathrm{~J}-\mathrm{W}$ Kim, ${ }^{1} \mathrm{NH}$ Park, ${ }^{1} \mathrm{Y}$-S Song, ${ }^{1} \mathrm{HS}$ Kim. ' ${ }^{1}$ Seoul National University College of Medicine, Department of Obstetrics and Gynecology, Seoul, Korea, Republic of; ' ${ }^{S}$ Seoul National University College of Medicine, Department of Radiology, Seoul, Korea, Republic of

\subsection{6/ijgc-2021-IGCS.288}

Objectives To evaluate the prognostic significance of residual tumor size on computed tomography (CT) after upfront surgery for advanced ovarian cancer (AOC).

Methods We collected data of patients with stage III-IV highgrade serous carcinoma of the ovary (HGSC) who underwent optimal cytoreduction between 2013 and 2018. They took CT between upfront surgery and adjuvant chemotherapy. We evaluated surgical and radiological residual tumor size after upfront surgery, which was divided into R0 (no residual lesion) and R1 (residual tumor $<1 \mathrm{~cm}$ ).

Results A total of 106 patients received surgical R0 $(n=73$, $68.9 \%)$ and R1 $(\mathrm{n}=33,31.1 \%)$. Among all patients, 66 $(62.3 \%)$ and $40(37.7 \%)$ showed radiologic R0 and R1, respectively. In 73 patients with surgical R0, 56 (76.7\%) and $17(23.3 \%)$ showed radiologic R0 and R1, whereas 10 $(30.3 \%)$ and $23(69.7 \%)$ were observed in 33 with surgical $\mathrm{R} 1$, respectively. In terms of survival, both surgical R0 and radiological R0 showed better progression-free survival (PFS; 26 vs. 16 mons; 33 vs. 15 mons; $p<0.05$ ), whereas no difference in overall survival based on residual tumor size. In multivariate analysis, surgical R0 was the only factor that improved PFS (adjusted HR, 0.45; 95\% CI, 0.21-0.98). On the other hand, radiologic R0 didn't reach statistical significance (adjusted HR, 0.58; 95\% CI, 0.14-1.03).

Conclusions Although patients with radiologic R0 showed better PFS in univariate analysis, there was no significance in multivariate analysis. Therefore, surgical R0 was more important factor to predict the prognosis of disease than radiologic R0 in AOC patients with optimal cytoreduction.

\section{EPV218/\#551 DEVELOPMENT OF A NOMOGRAM TO PREDICT THE FEASIBILITY OF MINIMALLY INVASIVE INTERVAL DEBULKING SURGERY IN PATIENTS WITH ADVANCED OVARIAN CANCER: A LARGE MONOCENTRIC COHORT STUDY}

${ }^{1} \mathrm{C}$ Conte*, ${ }^{1} \mathrm{~A}$ Rosati, ${ }^{1} \mathrm{C}$ Marchetti, ${ }^{1} \mathrm{~V}$ lacobelli, ${ }^{2} \mathrm{~V}$ Tranquillo, ${ }^{1} \mathrm{~S}$ Gueli Alletti, ${ }^{3} \mathrm{G}$ Scambia, ${ }^{3}$ A Fagotti. ${ }^{1}$ Fondazione Policlinico Universitario Agostino Gemelli, IRCCS, Gynecologic Oncology, Rome, Italy; ${ }^{2}$ Università cattolica del Sacro Cuore, Department of Woman and Child Health and Public Health, Roma, Italy; ${ }^{3}$ Università Cattolica del Sacro Cuore, Department of Woman and Child Health and Public Health, Woman Health Area, Fondazione Policlinico Universitario A. Gemelli Irccs, Roma, Italy

\subsection{6/ijgc-2021-IGCS.289}

Objectives Currently, no clear guidance defining the ideal candidate for minimally invasive interval debulking surgery (MIIDS) exists. This study aimed to identify predictive factors of minimally invasive approach feasibility in advanced ovarian cancer (AOC) patients who were candidates to IDS after neoadjuvant chemotherapy (NACT).

Methods This was a single institution, retrospective study. Perioperative variables were used to predict the likelihood of MIIDS using multivariable models. A nomogram was developed, and internal validation was performed using the bootstrapping correction technique.

Results Between 2014 and 2020, 108 (28.4\%) and 272 (71.6\%) patients underwent IDS by minimally invasive and open approach, respectively. Surgeon's expertise (OR:6.27, 95\% CI:3.25-12.08, p $\leq 0.001$ ), absence of omental cake (OR: 8.56, 95\% CI: 4.22-17.33, $\mathrm{p} \leq 0.001), \quad<2$ peritoneal sites involvement (OR:3.11, 95\% CI:1.45-6.65, p=0.003) and complete serological response (OR:2.23, 95\% CI:1.21-4.11, $\mathrm{p}=0.010)$ appeared to be significantly correlated with MI-IDS feasibility at multivariate analysis.

A nomogram was built to visualize the effect of perioperative variables on the estimated probability of MI-IDS in patients with a clinical response after NACT. We used the

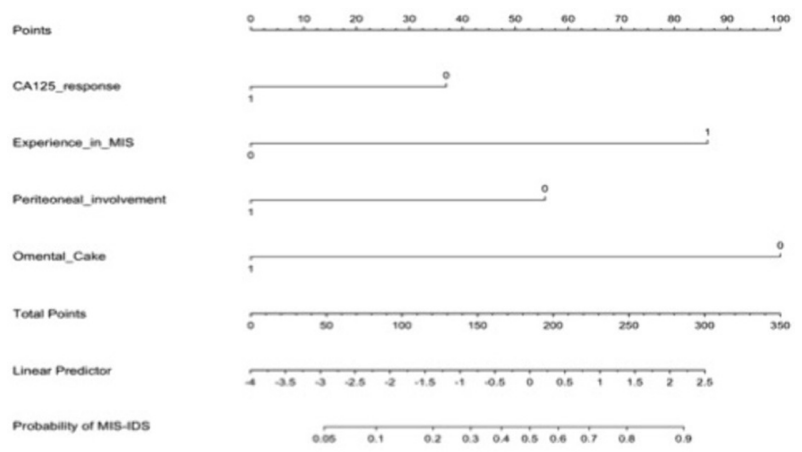

\section{Abstract EPV218/\#551 Figure 1}

C0125_response indicates complete response (0) or partial/stable serologic response (1). Experience_in_MIS indicates good surgeon's experience in MIS (1) or not (0). Pentoneal_involvement indicates $\geq 2$ sites ( 1 of $<2$ sites $(0)$ involved. Omental_cake indicates presence (1) or absence $(0)$ of oemental cake.

To use, find Ca125 response om Ca125_response axes, then draw straight line upward to points axis to determine how many points patient receives for Ca125_response. Do this again for other axes, each time drawing straight line upward toward points axis. Sum points reveived for each variable and find sum on total points axis. Draw straight line down to probability of MIS-IDS axix to find find patient's probability of receiving MIs-IDS. 
Abstract EPV218/\#551 Table 1 Logistic regression for prediction of MI-IDS

\begin{tabular}{|c|c|c|c|c|}
\hline Variables & OR (95\% CD & P.ralues & OR (95\% CD & P-xalus \\
\hline 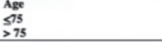 & $3.63(180.732)$ & ه..001 & $1.99(0.60-3.71)$ & 0.382 \\
\hline $\begin{array}{l}\text { BMII } \\
230 \\
230\end{array}$ & ${ }_{0.96}^{1}(0.56-1.67)$ & 0.904 & & \\
\hline $\begin{array}{l}\text { BRCA } \\
\text { Wild-ove } \\
\text { Mutatioo }\end{array}$ & $1.170 .63-2.16$ & 0.616 & & \\
\hline $\begin{array}{l}\mathrm{CCI} \\
>3 \\
>3\end{array}$ & $1.16(0.72-187)$ & 0.524 & & \\
\hline $\begin{array}{l}\text { Cal125 resposese } \\
\text { CR } \\
\text { PRSD }\end{array}$ & $186(1.14-3.30)$ & 0.012 & $2.23(1.21-4.11)$ & 0.010 \\
\hline $\begin{array}{l}\text { Mosisperience } \\
\text { No } \\
\text { Yes } \\
\text { Oos }\end{array}$ & $\begin{array}{c}1 \\
3.76(228-6.19)\end{array}$ & 8001 & $627025.1208)$ & $\$ .001$ \\
\hline $\begin{array}{l}\text { No } \\
\text { Nos } \\
\text { Yos }\end{array}$ & $1123(6.35-19.86)$ & $\infty .001$ & $856(4.22 \cdot 17.33)$ & 80.001 \\
\hline $\begin{array}{l}\text { Peritoneal sites involvement } \\
\text { i2 } \\
\geqslant 2\end{array}$ & $504(258.986)$ & $\infty .001$ & $3.11(1.45-6.65)$ & 0.003 \\
\hline
\end{tabular}

Abbreviations: BMI: body mass index; CCl: Charlson Comorbididty Index; CR: complete response; PR: partial response; $S D=$ stable disease

four significant perioperative variables according to logistic regression. The calibration plot demonstrated good agreement between the predicted and actual probability of MI-IDS $(\mathrm{p}=0.93$, Hosmer-Lemeshow test $)$.

Conclusions A nomogram might represent a useful tool to choose the best surgical approach in patients with AOC undergoing IDS.

\section{EPV219/\#581 FULL SYSTEMATIC LYMPHADENECTOMY FOR APPARENT EARLY STAGE OVARIAN CANCER: IMPACT ON SPECIFIC LYMPHATIC MORBIDITY}

E Ervas*, M Longo, A Lembo, V Artuso, A Di Siena, F Ghezzi, J Casarin. Women's and children hospital F. Del Ponte Hospital - University of Insubria, Obstetrics and Gynecology, varese, Italy

\subsection{6/ijgc-2021-IGCS.290}

Objectives To evaluate the rate of lymphatic-related morbidity among patients undergoing surgical staging for apparent earlystage ovarian cancer (EOC) and to report the specific patients' lymphatic complications.

Methods Data of consecutive patients who underwent surgical staging for EOC between 01/2002 and 12/2018 were analyzed. A self-reported validated 13-item lymphedema screening questionnaire was sent to evaluate specific lymphatic complications. Patients were stratified by the performance retroperitoneal staging into two groups: fully pelvic and aortic lymphadenectomy performed (LND) vs. no retroperitoneal staging (NO-LND). Patients who had conservative treatment were included in the study. The analysis focused only on women who answered the specific questionnaire. Patients lost

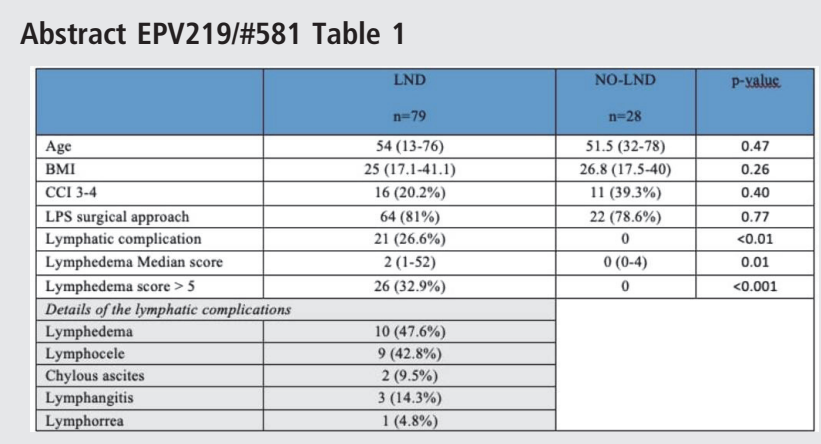

at follow-up and those who reported peripheral vascular disease at the time of surgery were excluded.

Results During the study period 140 patients were treated; according to the inclusion/exclusion criteria 107 represented our study population. Baseline characteristics such as age, BMI, Charlson Comorbidity Index (CCI) and surgical approach did not significantly differ between the groups. Patients in LND group (compared to NO-LND) had a higher rate of specific lymphatic complications $(26.6 \%$ vs. $0 \%, \mathrm{p}<0.01)$. The performance of lymphadenectomy significantly impacted the subjective lymphatic-related morbidity (score $>5$ ).

Conclusions Our study confirms a high correlation between the performance of LND and specific lymphatic morbidity in patients undergoing surgical staging for EOC. The dedicated 13 -item screening questionnaire might be a useful tool to categorize patients' perception of lymphatic-related complications, including lower extremity lymphedema.

\section{EPV220/\#594 MESONEPHRIC-LIKE MULLERIAN ADENOCARCINOMA OF THE OVARY}

${ }^{1} \mathrm{~A}$ Michas*, ${ }^{2} \mathrm{~T}$ Akrivos, ${ }^{2} \mathrm{P}$ Giannakas, ${ }^{1} \mathrm{E}$ Arvanitou, ${ }^{1} \mathrm{~K}$ Gkikas, ${ }^{1} \mathrm{~A}$ Kolomitrousi, ${ }^{1} \mathrm{C}$ Kagkaras, ${ }^{1} \mathrm{M}$ Gkiaouraki, ${ }^{1} \mathrm{~K}$ Mpalasis, ${ }^{1} \mathrm{C}$ Christofilakis, ${ }^{1} \mathrm{~N}$ Tsoukalas. ${ }^{1} 401 \mathrm{GMHA}$, Oncology, Athens, Greece; ${ }^{2} 401$ GMHA, Gynecology, Athens, Greece

\subsection{6/ijgc-2021-IGCS.291}

Objectives INTRODUCTION: Mesonephric-like Mullerian adenocarcinomas of the ovaries are extremely rare gynecological malignancies. Their embryological and histological origin remains debatable. The more prevalent tumorigenic theories support either development from Mesonephric duct remnants of the female genital tract, or development from Mullerian lesions that undergo Mesonephric differentiation.

Methods Case-report of a patient with ovarian Mesonephriclike Mullerian adenocarcinoma.

Results CASE REPORT: 64 years old female patient with medical history of hypothyroidism and dyslipidemia. During annual gynecological US-screening examination, a solid formation ( $4 \mathrm{~cm}$ diameter) was found on the left ovary. An ensuing MRI tomography diagnosed a solid ovarian mass. Further staging with CTs and PET-CT scan excluded distant neoplasmatic dissemination. Subsequently, surgical total hysterectomy was performed. After histological evaluation, the analysis concluded to low-grade Mesonephric-like Mullerian adenocarcinoma of left ovary, adjacent to multiple foci of endometriosis. Due to tumor rarity, the histological results were re-checked and verified by multiple histology experts. The patient received adjuvant chemotherapy with 6 circles of Carboplatin/paclitaxel. Treatment was completed without significant side-effects, except for mild nausea and hand-foot syndrome. Follow-up examinations showed complete disease-remission. Currently, the patient is regularly monitored with scheduled-periodic assessments, without any sign of recurrence. Moreover molecular analysis revealed heterozygous somatic KRAS mutation NM_033360.4:c.35G>A:p.(Gly12Asp), and heterozygous genomic PMS2 mutation NM_000535.7:c.2559C > G p.(Ile853Met)

Conclusions CONCLUSION: Mesonephric-like Mullerian adenocarcinomas of ovaries are extremely rare tumors $(<15$ literature reports in Pubmed, Scopus). The most prevalent theory of tumorigenesis, involves cancer development from Mullerian lesions (eg foci of endometriosis) that undergo Mesonephric differentiation. Further research is necessary to illuminate the neoplastic nature of these lesions. 